\title{
Central and peripheral effects of arecoline in patients with autonomic failure
}

\author{
Ronald J Polinsky, Robert T Brown, $M$ Teresa Curras, Susan M Baser, \\ Celeste E Baucom, Douglas R Hooper, Ann M Marini
}

\begin{abstract}
Increased plasma adrenalin (A) levels following arecoline in normal subjects and patients with multiple system atrophy (MSA) may result from nicotinic adrenal stimulation. Lack of this response in patients with pure autonomic failure (PAF) is consistent with peripheral sympathetic dysfunction. The mechanisms underlying diminished plasma corticotropin (ACTH) responses to arecoline may differ in patients with autonomic failure. Hypothalamic, cholinergic degeneration could prevent the response in MSA whereas patients with PAF do not manifest the normal increase in $A$ which may be required to elicit an ACTH response. The appearance and exacerbation of tremor, vertigo, and pathological affect in the MSA group suggest that some central cholinergic receptors remain functional.
\end{abstract}

Central and peripheral lesions of the autonomic nervous system result in failure to regulate vegetative functions in the body. The biochemical and pharmacological consequences of autonomic failure differ according to the nature and site of the lesion(s). ${ }^{1}$ Two clinical syndromes have been intensively investigated. Pure autonomic failure (PAF) occurs in the absence of peripheral neuropathy or signs of any central neurological disturbance. ${ }^{2}$ Low plasma noradrenalin (NA) levels, ${ }^{3}$ noradrenergic receptor supersensitivity, ${ }^{4}$ reduced neuronal NA stores, ${ }^{4}$ and diminished neuronal uptake of $\mathrm{NA}^{5}$ reflect the primary involvement of postganglionic sympathetic neurons in PAF. Multiple system atrophy (MSA) is a degenerative neurological disorder characterised clinically by autonomic failure, Parkinsonism, cerebellar dysfunction and pyramidal tract involvement. ${ }^{6}$ Indices of peripheral sympathetic neuronal function are normal in MSA. ${ }^{1}$ Several central nervous system neurotransmitter systems, however, are affected by the degenerative process. ${ }^{7-9}$

Hypoglycaemia is an effective stimulus for releasing an array of neurotransmitters, hormones, and peptides. Corticotropin (ACTH) and $\beta$-endorphin levels in plasma do not increase normally during hypoglycaemia in patients with MSA. ${ }^{10}$ Since release of ACTH is stimulated by centrally active cholinergic agonists such as arecoline, ${ }^{11}$ the diminished ACTH response to hypoglycaemia in MSA may reflect a hypothalamic cholinergic defect.
Alternatively, the mechanisms may be separate. In this study we measured catecholamine and ACTH responses to arecoline after glycopyrrolate administration in patients with autonomic failure and normal subjects to assess peripheral and central effects of cholinergic stimulation.

\section{Materials and methods}

Subjects. All subjects were admitted to the Clinical Center, National Institutes of Health (NIH), for the study. Medical history, neurological examination, electrocardiogram and blood studies were performed before the infusion test. A clinical diagnosis of MSA was made as described previously. ${ }^{4}$ Ten control subjects (seven men, three women) volunteered through the NIH normal volunteer programme. The mean (SEM) age for control subjects [58.5 (4) years] did not differ from the 15 patients (eight men, seven women) with MSA [57.9 (1.8) years] or those (two men, four women) with PAF [52.3 (1.8) years]. Medications were discontinued for at least one week before the study. There were no dietary restrictions. All subjects gave informed consent according to NIH guidelines.

Arecoline Test. All studies were performed in the morning following an overnight fast. An intravenous needle was inserted into a vein of one arm for sampling blood; a catheter placed in a vein of the opposite arm was kept patent with an infusion of normal saline. Subjects remained in the supine position throughout the test which was performed as previously described. ${ }^{11}$ Glycopyrrolate $(0.2 \mathrm{mg})$ was given intravenously to block peripheral muscarinic effects. After a 20 minute baseline period, subjects received arecoline hydrobromide (3 $\mathrm{mg}$ of base) intravenously over 10 minutes. Blood samples for measurement of catecholamines and ACTH were drawn just before arecoline administration, and at 10 minute intervals for the next hour. All samples were kept on ice and centrifuged immediately following the test. The plasma was removed and kept frozen at $-70^{\circ} \mathrm{C}$ until the time of assay. Blood pressure was measured every 10 minutes with a Dinamap $^{(i)}$ Vital Signs Monitor (Critikon, Tampa, FL).

Catecholamine Assay. Blood was drawn into tubes containing heparin. The catecholamines were partially purified and concentrated from plasma using alumina adsorption. Plasma adrenalin (A) and noradrenalin (NA) levels were determined using high-performance 
liquid chromatography (HPLC) with electrochemical detection. After thawing and centrifugation, $1 \mathrm{ml}$ of plasma was added to $400 \mu \mathrm{l}$ of Tris-EDTA buffer, $\mathrm{pH} 8 \cdot 6 ; 0.5 \mathrm{ng}$ of dihydroxybenzylamine was added as an internal standard. Approximately $10 \mathrm{mg}$ of acidwashed alumina was added; the tubes were centrifuged and the supernatants removed following 20 minutes of mixing. After the alumina was twice washed with water, catecholamines were eluted by vortexing the alumina with $200 \mu$ l of $0 \cdot 2 \mathrm{~N}$ acetic acid.

Catecholamines were separated in the eluate using a Microbondapak C18 reverse-phase column (Waters Associates, Milford, MA). The mobile phase consisted of sodium acetate, EDTA, heptanesulfonic acid, and acetonitrile; a flow rate of $1 \mathrm{ml} / \mathrm{min}$ was employed. Potentials of $0.3,0.3$, and $-0.3 \mathrm{~V}$ were applied respectively to the guard cell and two flowthrough glassy carbon electrodes connected in series. The coulometric detector was set at a gain of 8000 . Plasma NA and A levels were calculated from peak height after correction for recovery (approximately 70\%). Approximately $15 \mathrm{pg} / \mathrm{ml}$ of plasma catecholamines could be detected easily with this method. Interassay variation was less than $12 \%$.

Measurement of $A C T H$. Blood ( $2 \mathrm{ml}$ ) was collected into chilled tubes containing potassium EDTA $(10.5 \mathrm{mg})$ and aprotinin (1000 KIU). The plasma was removed after centrifugation and frozen at $-70^{\circ} \mathrm{C}$ until performance of the ACTH assay with a radioimmunoassay kit (Incstar, Stillwater, MN). Samples and standards were first thawed on crushed ice; then $200 \mu \mathrm{l}$ of each was incubated in polystyrene tubes for 24 hours at $4^{\circ} \mathrm{C}$ with $200 \mu \mathrm{l}$ of rabbit anti-ACTH serum. After addition of $100 \mu \mathrm{l}$ of ${ }^{125} \mathrm{I}-\mathrm{ACTH}$, samples were incubated an additional 24 hours at $4^{\circ} \mathrm{C}$. Goat anti-rabbit precipitating complex $(500 \mu \mathrm{l})$ was then added, and incubation for 20 minutes at $22^{\circ} \mathrm{C}$ was performed. Tubes were finally centrifuged using $760 \mathrm{~g}$ at $4^{\circ} \mathrm{C}$, the supernatant was aspirated and the precipitates were counted in a gamma counter. Standards were assayed in triplicate, and samples in duplicate. This procedure yielded a lower limit of detection $\left(95 \% \mathrm{~B} / \mathrm{B}_{0}\right)$ of $7 \cdot 3 \mathrm{pg} / \mathrm{ml}$ with an interassay variation of approximately $13 \%$.

Data Analysis. The plasma ACTH and catecholamine responses were calculated for each subject by subtracting the baseline from the levels at each time point. Mean blood pressure (MBP) was calculated as diastolic BP plus one-third of pulse pressure. Comparison between control subjects and patients with
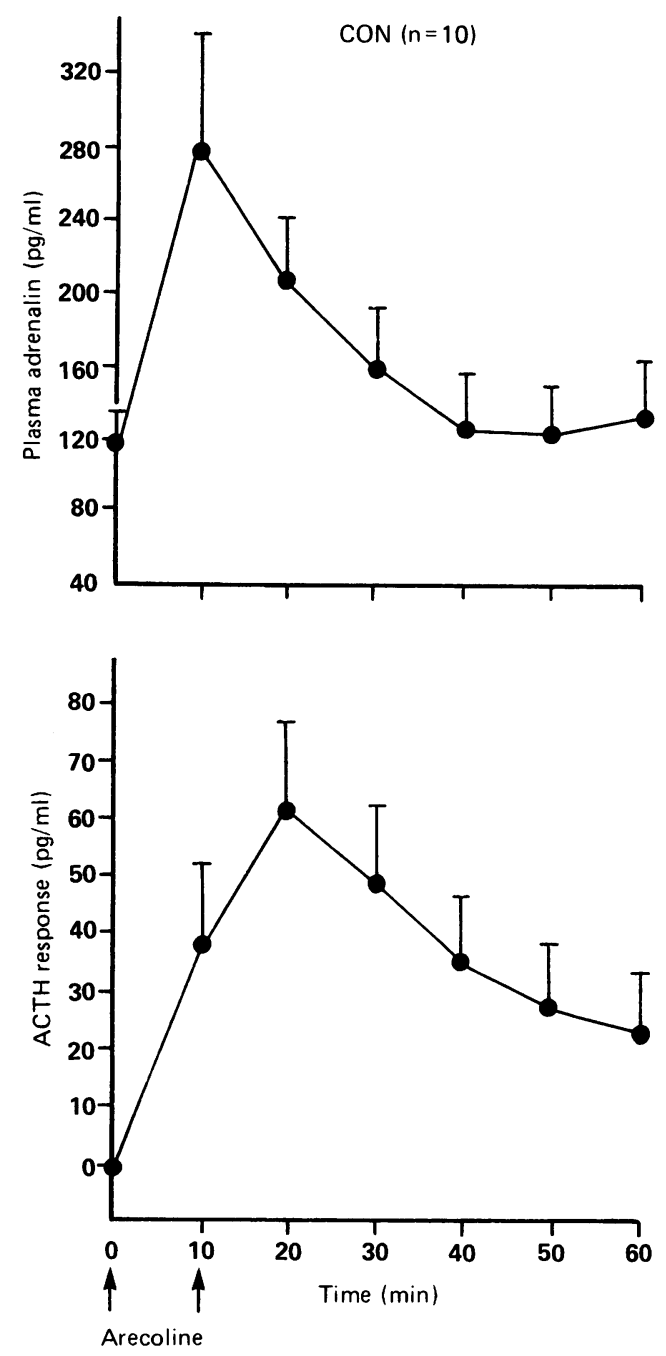

Figure 1 Plasma adrenalin and ACTH responses to arecoline administration in normal subjects.

autonomic failure was performed by analysis of variance with appropriate follow up tests. The relationship between ACTH and catecholamines was assessed by linear regression. A $2 \times 2$ Chi squared test of homogeneity was used to compare the frequency of occurrence of various signs and symptoms.

\section{Results}

Baseline ACTH levels did not differ among the three groups of subjects. As shown previously, ${ }^{34}$ the supine plasma NA level was significantly $(p<0.05)$ lower in patients with PAF than in normal subjects or MSA patients. Although glycopyrrolate did not affect baseline plasma levels of ACTH or NA, the drug did significantly $(p<0.01)$ increase plasma $A$ in all

Table 1 The effect of glycopyrrolate on plasma catecholamines and ACTH in normal subjects and patients with autonomic failure. Mean (SEM)

\begin{tabular}{|c|c|c|c|c|c|c|c|c|c|c|c|}
\hline \multirow{3}{*}{$\begin{array}{l}\text { Diagnosis } \\
\text { CON } \\
\text { MSA } \\
\text { PAF }\end{array}$} & \multicolumn{4}{|c|}{ Noradrenaline (pg/ml) } & \multicolumn{3}{|c|}{ Adrenalin (pg/ml) } & \multicolumn{4}{|c|}{$A C T H(p g / m l)$} \\
\hline & \multicolumn{2}{|c|}{ Baseline } & \multicolumn{2}{|c|}{ Glycopyrrolate } & \multirow{2}{*}{$\begin{array}{l}\text { Baseline } \\
20 \\
20 \\
20\end{array}$} & \multicolumn{2}{|c|}{ Glycopyrrolate } & \multicolumn{2}{|c|}{ Baseline } & \multicolumn{2}{|c|}{ Glycopyrrolate } \\
\hline & $\begin{array}{r}225 \cdot 3 \\
305 \cdot 1 \\
72 \cdot 4\end{array}$ & $\begin{array}{l}(21 \cdot 1) \\
(92 \cdot 8) \\
(20 \cdot 4)^{\star}\end{array}$ & $\begin{array}{l}331 \cdot 3 \\
267 \cdot 8 \\
200 \cdot 1\end{array}$ & $\begin{array}{l}(53.3) \\
(55.3) \\
(70.0)\end{array}$ & & $\begin{array}{r}126.9 \\
117 \cdot 7 \\
66.6\end{array}$ & $\begin{array}{c}(15 \cdot 3) \dagger \\
(31 \cdot 8) \ddagger \\
(5 \cdot 0) \dagger\end{array}$ & $\begin{array}{l}19 \cdot 9 \\
17 \cdot 1 \\
13 \cdot 1\end{array}$ & $\begin{array}{l}(4 \cdot 1) \\
(2 \cdot 6) \\
(2 \cdot 1)\end{array}$ & $\begin{array}{l}21.5 \\
19 \cdot 8 \\
16 \cdot 2\end{array}$ & $\begin{array}{l}(4 \cdot 3) \\
(2 \cdot 0) \\
(3 \cdot 0)\end{array}$ \\
\hline
\end{tabular}

${ }^{\star} \mathrm{p}<0.05$, compared to control subjects; $\nmid \mathrm{p}<0.005$, compared to baseline; $\ddagger \mathrm{p}<0.01$, compared to baseline. 
Figure 2 Plasma $A C T H$ responses to arecoline in normal subjects and patients with autonomic failure.

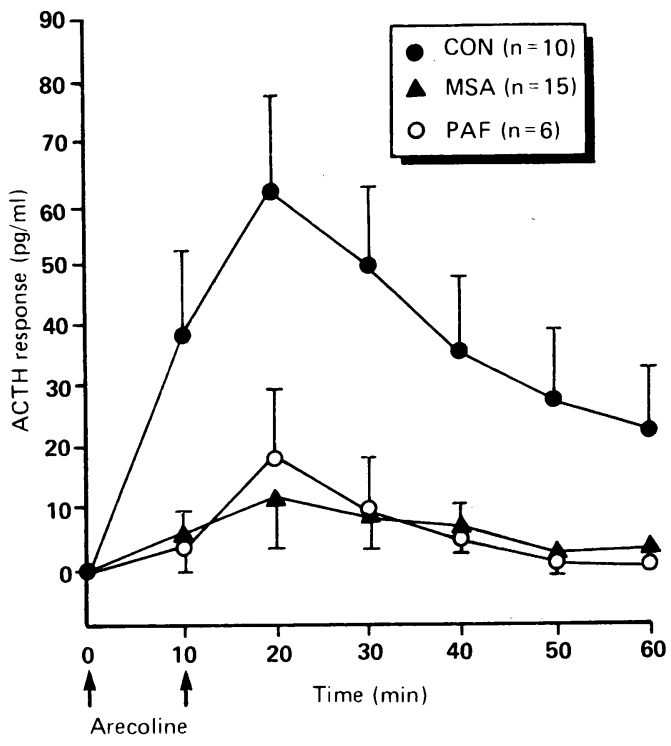

groups (table 1). We have previously observed an increase in plasma A levels beginning 10 minutes after glycopyrrolate administration; the increment in plasma A levels remained constant and was sustained for approximately 150 minutes after which the levels return to baseline (unpublished observations). In normal subjects, arecoline administration was followed by increases in plasma A and ACTH which were maximal at 10 and 20 minutes respectively (fig 1 ). The plasma ACTH response was virtually absent in MSA; a slight increase was observed in patients with PAF. However, the maximal increment in plasma

Figure 3 Mean (SEM) baseline and maximum plasma catecholamine levels following arecoline administration in normal subjects and patients with $M S A$ and $P A F$.

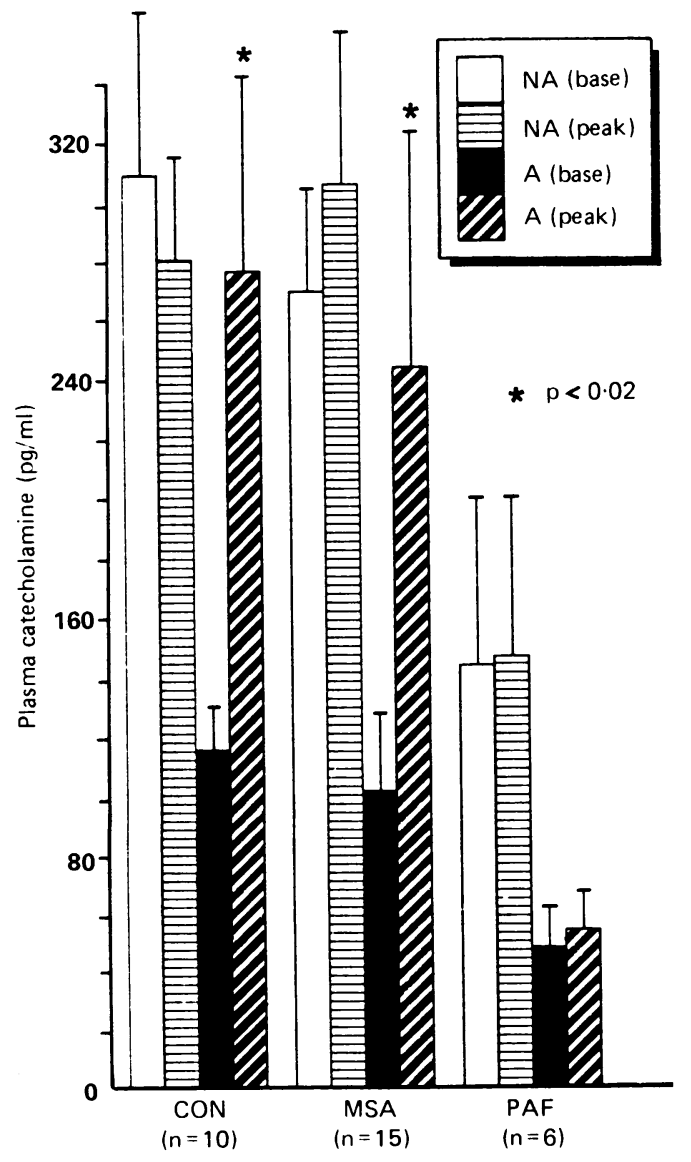

Table 2 Cardiovascular responses to arecoline in normal subjects and patients with autonomic failure. Mean (SEM)

\begin{tabular}{|c|c|c|c|}
\hline \multirow{2}{*}{$\begin{array}{l}\text { Diagnosis } \\
\text { CON } \\
\text { MSA } \\
\text { PAF }\end{array}$} & $\begin{array}{l}\text { MBP increment } \\
(\mathrm{mm} \mathrm{Hg})\end{array}$ & \multicolumn{2}{|c|}{$\begin{array}{l}\text { HR increment } \\
(\text { bpm })\end{array}$} \\
\hline & $\begin{aligned} 11.2 & (3.0)^{\star} \\
11.3 & (2.5)^{\dagger} \\
3.5 & (8.5)\end{aligned}$ & $\begin{array}{r}28.9 \\
8.4 \\
9.8\end{array}$ & $\begin{array}{l}(3 \cdot 9) \dagger \\
(1 \cdot 7)^{\dagger} \\
(6 \cdot 2)\end{array}$ \\
\hline
\end{tabular}

${ }^{\star} \mathrm{p}<0.005 ; \mathrm{tp}<0.001$

ACTH was significantly $(\mathrm{p}<0.01)$ lower than normal in patients with MSA and PAF (fig 2). The magnitude of the plasma ACTH response in MSA was not related to previous anticholinergic therapy.

Plasma A levels were significantly $(\mathrm{p}<0.02)$ higher following arecoline only in normal subjects and patients with MSA (fig 3). None of the groups manifested an increase in plasma NA. There was a significant correlation $(r=0.61, p<0.02)$ between the increments in plasma ACTH and $A$ in response to arecoline in normal subjects and patients with PAF.

MBP increased by 11.2 (3) and 11.3 (2.5) $\mathrm{mm} \mathrm{Hg}$ in normal subjects and patients with MSA; in PAF, there was not a significant change in blood pressure (table 2). Concomitant with the pressor response in normal subjects was a significant elevation in heart rate. A smaller, significant increase in heart rate occurred in the MSA patients. Although there was a similar increase in PAF, the change was not statistically significant.

Mild vertigo, nystagmus and nausea occurred in several subjects in each group. Onset or exacerbation of tremor was observed in seven MSA patients, but in none of the controls or patients with PAF. Several subjects in each group expressed a subjective alteration in mood but denied any emotional concomitant of their experience. In some patients with MSA, episodes of extreme, uncontrollable laughing or crying developed. No similar symptoms appeared during the baseline period or the last 40 minutes of the test. Only tremor and pathological affective changes were significantly $(\mathrm{p}<0.05)$ more frequent in patients with MSA. All symptoms resolved in all subjects within 10 minutes following cessation of drug administration.

\section{Discussion}

The ACTH response to cholinergic stimulation is impaired in patients with autonomic failure. However, the mechanism underlying this neuroendocrine abnormality may differ in patients with MSA and PAF. Release of anterior pituitary hormones is primarily controlled by hypothalamic releasing factors. ${ }^{12}$ Several neurotransmitter systems also modulate pituitary responses to physiological and pharmacological stimuli. The cholinergic and noradrenergic systems are particularly important with respect to ACTH release. Acetylcholine stimulates the release of corticotropin releasing factor (CRF) from hypothalamic synaptosomes. ${ }^{13}$ Although arecoline 
has muscarinic ${ }^{14}$ and nicotinic ${ }^{1516}$ effects, the presence of nicotinic receptors in the hypothalamus ${ }^{17}$ suggests that a nicotinic mechanism may be responsible for ACTH release. Nicotine administered into the third ventricle increases ACTH. ${ }^{18}$ Blockade of this effect with a central but not peripheral nicotinic antagonist, ${ }^{18}$ and the lack of CRF release following administration of bethanecol, ${ }^{19}$ a pure muscarinic agonist, add further support for a central nicotinic cholinergic pathway mediating ACTH release.

Catecholamine effects on ACTH are more complex. Although catecholamines can enhance the release of ACTH by CRF, ${ }^{20}$ acetylcholine-stimulated CRF activity is reduced by NA. Locus ceruleus activation also decreases $\mathrm{CRF}$ release. ${ }^{21}$ In contrast, peripheral administration of isoproterenol causes ACTH levels to rise, even in rats whose pituitary has been separated from the brain by stalk sectioning or lesioning of the median eminence..$^{22}$ Release of ACTH during insulin-induced hypoglycaemia can be blocked by propranolol. ${ }^{23}$ Thus it appears that adrenal medullary release of $\mathbf{A}$ may be required to elicit the ACTH response. However, normal ACTH and $\beta$-endorphin responses to hypoglycaemia occur in patients with PAF who have adrenergic insufficiency. ${ }^{10}$

In this study ACTH and NA did not increase normally following arecoline administration in patients with MSA and PAF, but plasma A responses differed in these disorders. Although plasma A levels increased following glycopyrrolate administration to patients with PAF, the increment was less than in normal subjects or patients with MSA. It is likely that peripheral involvement in PAF is not complete, as indicated by the preservation of pressor and NA responses to tyramine. ${ }^{4}$ Increased plasma A levels to arecoline in normal subjects and patients with MSA may result from nicotinic stimulation of the adrenal medulla since muscarinic effects of the drug were blocked by glycopyrrolate. Lack of this response and the low, resting plasma NA levels in PAF are also consistent with peripheral sympathetic involvement. These results differ from the ACTH and catecholamine responses observed during insulin-induced hypoglycaemia in patients with autonomic failure. Most patients with MSA or PAF have deficient catecholamine responses, ${ }^{24}$ whereas the increment in ACTH and $\beta$-endorphin is considerably diminished or absent only in those with MSA. $^{10} \mathrm{~A}$ lesion at any point in the sympathetic pathways can block the adrenal medullary response to hypoglycaemia in contrast to the effects of direct stimulation with a cholinergic agonist which would only be affected by peripheral dysfunction.

The interaction between central and peripheral cholinergic effects in mediating ACTH release provides a basis for formulating a hypothesis to explain the abnormal responses in patients with autonomic failure. Arecoline increases plasma $\beta$-endorphin even when peripheral muscarinic effects are blocked; ${ }^{11}$ atropine prevents the rise in $\beta$-endorphin during hypoglycaemia. ${ }^{25}$ Thus it appears that a central cholinergic pathway effects these peptide responses to hypoglycaemia. Studies in animals suggest, however, that a peripheral catecholamine response, specifically secretion of $A$ from the adrenal medulla, also mediates release of ACTH. Adrenalin can cross the blood-brain barrier in the region of the hypothalamus in cats. ${ }^{26}$ Failure of ACTH to rise in response to hypoglycaemia or arecoline in MSA probably reflects dysfunction of cholinergic-stimulated CRF release since the plasma $A$ responses to arecoline were normal. Although an exaggerated ACTH response might be anticipated as a manifestation of central cholinergic supersensitivity, this did not occur in patients with MSA.It may be that post-synaptic elements are affected in a long-standing degenerative disorder or supersensitivity results in depolarisation blockade leading to reduced ACTH secretion. Hypothalamic degeneration in MSA is attended by a decrease in the level of choline acetyltransferase, ${ }^{27}$ a marker of cholinergic neuronal integrity. We previously reported low CSF levels of acetylcholinesterase in patients with MSA, ${ }^{9}$ consistent with central cholinergic involvement. As discussed earlier, ACTH release is modulated through a variety of mechanisms. Thus it is not surprising that basal levels are normal despite cholinergic degeneration in MSA.

ACTH levels do not increase following arecoline administration in PAF, perhaps because they do not manifest the normal increase in plasma $A$ which may be required to elicit this response. The importance of this adrenergic-cholinergic interaction is highlighted by the correlation between the increments in plasma ACTH and A following arecoline administration. The catecholamine responses to hypoglycaemia are not completely absent in PAF. ${ }^{1024}$ Normal ACTH and $\beta$-endorphin responses to hypoglycaemia in PAF may occur because these patients develop $\alpha^{-4}$ and $\beta$ adrenergic $^{2829}$ receptor supersensitivity. The ACTH responses during hypoglycaemia may also be mediated through mechanisms that do not require substantial adrenal medullary activity.

Peripheral nicotinic stimulation of sympathetic ganglia or the adrenal medulla could cause the elevation of MBP induced by arecoline in normal subjects and patients with MSA since peripheral muscarinic effects were blocked. ${ }^{15}$ Furthermore, a central action of arecoline is to produce hypotension. ${ }^{30}$ An increase in circulating A levels generally results in a mild pressor effect since this catecholamine stimulates both classes of adrenergic receptors. The usual cardiovascular response to $\mathrm{A}$ administration is characterised by an increase in systolic, but decrease in diastolic blood pressure; the systolic change is usually greater than the effect on diastolic so that the MBP increases slightly. Since the elevation of MBP in these two groups was nearly identical in this study, it appears that this dose of arecoline is much greater than the threshold dose required for this response. The lack of a significant change in MBP in patients with PAF is consistent with their abnormal A responses to arecoline. Interpreta- 
tion of the arecoline effects on heart rate is more complex because there are direct cholinergic effects that oppose the chronotropic effect of $\beta$ adrenergic stimulation by $A$ in addition to reflex changes induced by alterations in MBP. Cardioaccelerator actions predominate in the normal subjects; the increase observed in the patient groups may be less since baroreflex function is compromised.

Vertigo, nausea, and nystagmus were experienced by a similar proportion of patients and normal subjects. This combination of symptoms may represent a pharmacologically induced sickness similar to that described for physostigmine. ${ }^{31}$ Motion sickness may result from a noradrenergic-cholinergic imbalance in the vestibular nuclei ${ }^{3233}$ and the cholinergic receptors involved are muscarinic. ${ }^{34}$ These side effects from arecoline could therefore result from stimulation of muscarinic receptors in the medial vestibular nuclei. There is no reason to suspect that this area is involved in these disorders. It is not surprising therefore that the symptoms occurred with similar frequency in all groups.

Exacerbation or onset of tremor occurred only in patients with MSA. Centrally active muscarinic antagonists are effective in reducing tremor in Parkinson's disease, or that induced by physostigmine. ${ }^{35}$ Hence, this arecoline effect may result from an increase in striatal cholinergic activity. Although the tremor was only seen in the MSA patients, this does not necessarily imply cholinergic supersensitivity since depletion of striatal dopamine may also be a factor.

Affective changes have been previously reported in normal subjects given higher doses of arecoline subcutaneously. ${ }^{36}$ In this study the appearance of this effect at a lower dose might be due to the intravenous route of administration. Dramatic emotional manifestations were observed in seven of the MSA patients, four of whom specifically denied any feelings consonant with their outward appearance. It appears that arecoline may have precipitated episodes of pathological affect that often occur as part of the clinical picture in MSA. This syndrome of pathological laughing or crying has been successfully treated in other diseases with levodopa, ${ }^{37}{ }^{38}$ or amantadine ${ }^{38}$ which would be consistent with dopaminergic deficiency as the basis for this phenomenon. Precipitation by arecoline and amelioration in other patients by amitriptyline, ${ }^{39}$ however, is also consistent with a role for relatively increased cholinergic activity in producing this symptom in MSA. Since the predominant lesion in many cases of pathological laughing and crying may involve the neostriate grey matter of the left hemisphere, ${ }^{40}$ a dopaminergic-cholinergic balance may therefore also exist in control of the motor system for emotional expression. The symptoms could result from a relative cholinergic predominance, analogous to the striatal imbalance in Parkinsonism. ${ }^{35}$

In summary, these results provide further distinction between PAF and MSA. The lack of an increment in plasma $A$ following arecoline administration in patients with PAF suggests that adrenal medullary innervation is affected by the peripheral involvement in this disorder. The resulting catecholamine deficiency may prevent a normal ACTH response to arecoline in PAF. Decreased catecholamine responses during hypoglycaemia in MSA may result from a central lesion(s) since $A$ levels rise in response to stimulation by a cholinergic agonist. The diminished ACTH response in MSA is consistent with a central abnormality involving a nicotinic cholinergic pathway. Pulmonary, vestibular and striatal responses during cholinergic stimulation suggest relative sparing of muscarinic receptors. This pattern of cholinergic involvement is similar to that described in Parkinson's disease. ${ }^{42}$

We are indebted to the many physicians in the United States who allowed their patients to participate in this study. Ms Linda E Nee provided valuable emotional support to many of the chronically ill patients. The staff of Ward 5-East, NIH Clinical Center, cared for the patients. Ms Jennifer $R$ Spahn assisted in the preparation of this manuscript.

1 Polinsky RJ. Clinical autonomic neuropharmacology. Clinical Neuropharmacology 1990;8:77-92

Bradbury S, Eggleston C. Postural hypotension: A report of hree cases. Am Heart J 1925;1:73-86.

3 Ziegler M, Lake C, Kopin I. The sympathetic nervous system defect in primary orthostatic hypotension. $N$ Engl J Med 1977;296:293-7.

4 Polinsky RJ, Kopin IJ, Ebert MH, Weise V. Pharmacologic distinction of different orthostatichypotension syndromes. Neurology 1981;31:1-7.

5 Polinsky RJ, Goldstein DS, Brown RT, Keiser HR, Kopin IJ. Decreased sympathetic neuronal uptake in idiopathic orthostatic hypotension. Ann Neurol 1985;18:48-53.

6 Shy GM, Drager GA. A neurological syndrome associated with orthostatic hypotension: A clinical-pathological with orthostatic hypotension: A

7 Polinsky RJ, Jimerson DC, Kopin IJ. Chronic autonomic failure: CSF and plasma 3-methoxy-4-hydroxyphenylfailure: CSF and plasma 3-meth
glycol. Neurology 1984;34:979-83.

8 Polinsky RJ, Brown RT, Burns RS, Harvey-White J, Kopin IJ. Low lumbar CSF levels of homovanillic acid and 5hydroxyindoleacetic acid in multiple'system atrophy with autonomic failure. J Neurol Neurosurg Psychiatry 1988; 51:914-19.

9 Polinsky RJ, Holmes KV, Brown RT, Weise V. CSF acetylcholinesterase levels are reduced in multiple system atrophy with autonomic failure. Neurology 1989;39:40-44.

10 Polinsky RJ, Brown RT, Lee GK, et al. Beta-endorphin $\mathrm{ACTH}$, and catecholamine responses in chronic autonomic failure. Ann Neurol 1987;21:573-7.

11 Risch SC, Kalin NH, Cohen RM, et al. Muscarinic cholinergic influences on ACTH and $\beta$-endorphin release mechan isms in human subjects. Peptides 1981;2(suppl 1):95-7.

12 Antoni FA. Hypothalamic control of adrenocorticotropin secretion: Advances since the discovery of 41-residue corticotropin-releasing factor. Endocrine Reviews 1986;7: corticotrop

13 Edwardson JA, Bennett GW. Modulation of corticotrophinreleasing factor release from hypothalamic synaptosomes. Nature 1974;251:425-7.

14 Baker RW, Chothia CH, Pauling P, Petcher TJ. Structure and activity of muscarinic stimulants. Nature 1971;230 439-45.

15 Euler US, Domeij B. Nicotine-like actions of arecoline. Acta Pharmacol 1945;1:263-9.

16 Riehl JL, Paul-David J, Unna KR. Comparison of the effects of arecoline and muscarine on the central nervous system. Int J Neuropharmacol 1962;1:393-401.

17 Wonnacott S. Brain nicotine binding sites. Human Toxicol 1987;6:343-53.

18 Matta SG, Beyer HS, McAllen KM, Sharp BM. Nicotine elevates rat plasma ACTH by a central mechanism. J Pharmacol Exp Ther 1987;243:217-26.

19 Hillhouse EW, Burden J, Jones MT. The effect of various putative neurotransmitters on the release of corticotrophin releasing hormone from the hypothalamus of the trophin releasing hormone from the hypothalamus of the rat in vitro. I. The effect of acetylch

20 Vale WW, Rivier C, Spiess J, Rivier J. Corticotropin releasing factor. In: Krieger DT, Brownstein MJ, Martin releasing factor. In: Krieger DT, Brownstein MJ, Martin JB, eds. Brain

21 Walker JM, Khachaturian H, Watson SJ. Some anatomical and physiological interactions among noradrenergic systems and opioid peptides. In: Ziegler MG, Lake CR, eds. Norepinephrine. Baltimore: Williams and Wilkins, 1984: 74-91.

22 Mezey E, Reisine TD, Palkovits $M$, et al. Direct stimulation of $\beta_{2}$-adrenergic receptors in rat anterior pituitary induces 
the release of adrenocorticotropin in vivo. Proc Natl Acad Sci USA 1983;80:6728-31.

23 Mezey E, Reisine TD, Brownstein MJ, et al. $\beta$-adrenergic mechanism of insulin-induced adrenocorticotropin release from the anterior pituitary. Science 1984;226: 1085-7.

24 Polinsky RJ, Kopin IJ, Ebert MH, Weise V. The adrena medullary response to hypoglycemia in patients with orthostatic hypotension. J Clin Endocrinol Metab 1980 51:1401-6.

25 Copolov D, Jethwa J, Stern A, et al. Insulin hypoglycemia and cholinergic blockade: response of plasma immunoreactive $\beta$-endorphin. Clin Endocrinol (Oxf) 1983;19: 575-80.

26 Weil-Malherbe H, Axelrod J, Tomchick R. Blood-brain barrier for adrenaline. Science 1959;129:1226-7.

27 Spokes E, Bannister R, Oppenheimer D. Multiple system atrophy with autonomic failure: clinical, histological, and atrophy with autonomic failure: clinical, histological, and neurochemical

28 Robertson D, Hollister A, Carey E, et al. Increased vascular $\beta_{2}$-adrenoceptor responsiveness in autonomic dysfunction. J Am Coll Cardiol 1984;3:850-6.

29 Baser SM, Curras MT, Baucom C, Brown RT, Polinsky RJ Cardiovascular responses to isoproterenol in patients with autonomic failure. Neurology 1989;39(Suppl 1):423

30 Porsius AJ, Mutschler E, Van Zwieten PA. The central action of various arecaidine esters (arecoline derivatives) on blood pressure and heart rate in the cat. Arzneim Forsch 1978;28:1373-6.

31 Janowsky DS, Risch SC, Ziegler M, Kennedy B, Huey L. A cholinomimetic model of motion sickness and space adaptation syndrome. Aviat Space Environ Med 1984;55: $692-6$

32 Wood CD, Graybiel A. Theory of antimotion sickness drug mechanisms. Aerospace Med 1972;43:249-52.

33 Wood CD. Antimotion sickness and antiemetic drugs. Drugs 1979;17:471-9.

34 Kohl RL, Homick JL. Motion sickness: A modulatory role for the central cholinergic nervous system. Neurosci Biobehav Rev 1983;7:73-85.

35 Duvoisin RC. Cholinergic-anticholinergic antagonism in parkinsonism. Arch Neurol 1967;17:124-36.

36 Nurnberger JI, Jimerson DC, Simmons-Alling S, et al. Behavioral, physiological and neuroendocrine responses to arecoline in normal twins and "well state" bipolar patients. Psychiatry Res 1983;9:191-200.

37 Wolf JK, Santana HB, Thorpy M. Treatment of "emotional incontinence" with levodopa. Neurology 1979;29:1435-6.

38 Udaka F, Yamao S, Nagata H, Nakamura S, Kameyama M. Pathologic laughing and crying treated with levodopa.

39 Schiffer RB, Herndon RM, Rudick RA. Treatment of pathologic laughing and weeping with amitriptyline. $N$ Engl J Med 1985;312:1480-2.

40 Poeck K. Pathophysiology of emotional disorders associated with brain damage. In: Vinken PJ, Bruyn GW, eds. Handbook of clinical neurology, Vol 3. Amsterdam: NorthHolland Publishing Company, 1969:343-67.

41 Brown RT, Polinsky RJ, Machado CE, Liberato DJ, Yergey AL. Ganglionic responsivensss to acetylcholine in autonomic failure. Neurology 1987;37(suppl 1):357.

42 Perry EK, Perry RH, Smith CJ, et al. Cholinergic receptors in cognitive disorders. Can J Neurol Sci 1986;13:521-7. 